\title{
First results of the newly installed, MAPS based, ALICE Inner Tracking System
}

\author{
Jian Liu ${ }^{a, *}$ on behalf of the ALICE Collaboration \\ ${ }^{a}$ University of Liverpool, \\ Department of Physics, Liverpool, United Kingdom \\ E-mail: j.1.liu@liverpool.ac.uk
}

The ALICE Inner Tracking System (ITS) has recently been replaced with a full silicon pixel detector constructed entirely with Complementary Metal-Oxide-Semiconductor (CMOS) monolithic active pixel sensors. It consists of three inner layers (50 $\mu \mathrm{m}$ thick sensors) and four outer layers (100 $\mu \mathrm{m}$ thick sensors) covering $10 \mathrm{~m}^{2}$ and containing 12.5 billion pixels with a pixel size of $27 \mu \mathrm{m} \times 29 \mu \mathrm{m}$. Its increased granularity, the very low material budget $\left(0.35 \% \mathrm{X}_{0} /\right.$ layer in the inner barrel) as well as a small radius of the innermost layer combined with a thin beam pipe, will result in a significant improvement of impact-parameter resolution and tracking efficiency at low transverse momentum with respect to the previous tracker.

The assembly of the full detector and services finished in December 2019. A comprehensive commissioning phase (on surface) was completed in December 2020, including detector calibration, fake-hit rate determination, data transmission tests, and preliminary evaluation of the detector efficiency and the alignment of the sensors, based on a reconstruction of cosmic-ray tracks. The commissioning of the new ITS within the ALICE apparatus has started in May 2021. After a first phase of standalone tests and detector performance optimization, the detector has been included in the global commissioning activities from July 2021. In this paper, the detector design as well as the first results of the performance studied during the commissioning of the detector will be discussed.

\footnotetext{
*** The European Physical Society Conference on High Energy Physics (EPS-HEP2021), ***

*** 26-30 July $2021 * * *$

*** Online conference, jointly organized by Universität Hamburg and the research center DESY ***
}

\footnotetext{
${ }^{*}$ Speaker
} 


\section{Introduction}

ALICE [1] (A Large Ion Collider Experiment) is a general-purpose detector dedicated to heavyion physics at the Large Hadron Collider (LHC). It aims to use nucleus-nucleus, proton-nucleus and proton-proton collisions to study the physics of the Quark-Gluon Plasma (QGP) and strongly interacting matter at extreme energy densities.

The ALICE detector is currently being upgraded during the LHC Long Shutdown 2 (LS2). An integrated luminosity of $13 \mathrm{nb}^{-1}$ will be delivered for $\mathrm{Pb}-\mathrm{Pb}$ collisions during the LHC Run 3 . The instantaneous luminosity will reach a level of $6 \times 10^{27} \mathrm{~cm}^{-2} \mathrm{~s}^{-1}$ resulting in a minimum-bias $\mathrm{Pb}-\mathrm{Pb}$ interaction rate of about $50 \mathrm{kHz}$, which is well beyond the specifications of the detector operated during the LHC Run 1 and Run 2.

One of the upgrades, driven by the requirements for the ALICE experiment in Run 3, is the construction of a new Inner Tracking System [2]: ITS2. The ITS2 will lead to a significant improvement of the tracking efficiency, in particular for the particles with transverse momentum smaller than $1 \mathrm{GeV} / c$. The design of the detector and the performance studied from the commissioning at the laboratory are discussed in Section 2 and 3.

\section{Detector Design, Production and Installation}

The upgraded Inner Tracking System, ITS2, consists of seven cylindrical layers as sketched in Fig. 1. The innermost three layers form the Inner Barrel (IB) and the outermost four layers, two Middle Layers (ML) and two Outer Layers (OL) compose the Outer Barrel (OB). The ITS2 is based

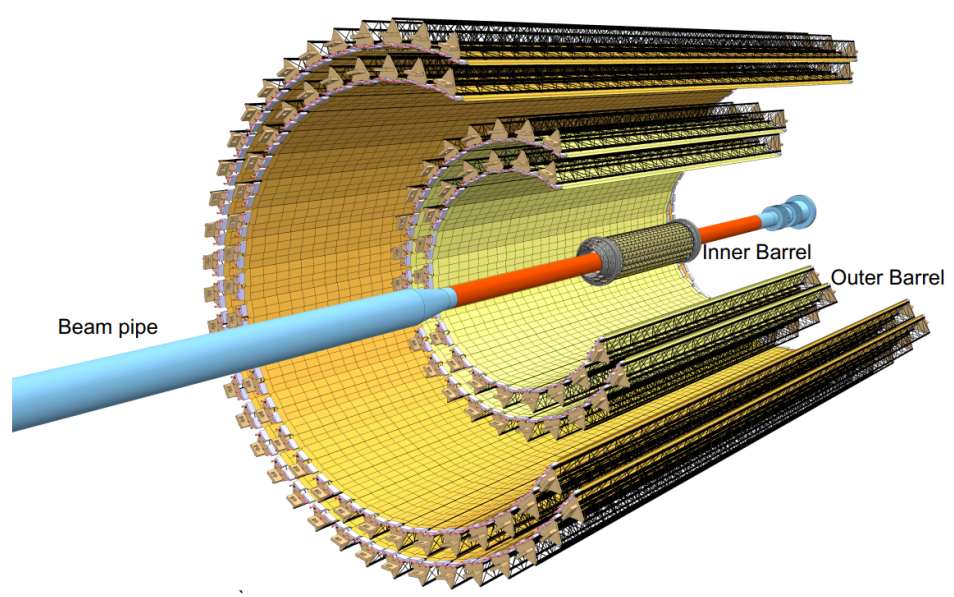

Figure 1: ITS2 layout. Inner Barrel: 3 Inner Layers. Outer Barrel: 2 Middle Layers and 2 Outer Layers.

on the Monolithic Active Pixel Sensor (MAPS) technology, and the pixel sensor is known as ALice PIxel DEtector (ALPIDE) [3].

The ALPIDE sensor is produced by using the TowerJazz $180 \mathrm{~nm}$ CMOS imaging sensor process. A high-resistivity $(1 \mathrm{k} \Omega \cdot \mathrm{cm})$ p-type epitaxial layer allowing $-3 \mathrm{~V}$ back-bias is chosen to improve the charge collection efficiency and the radiation hardness. It has demonstrated a radiation tolerance of $270 \mathrm{kRad}$ for Total Ionizing Dose (TID) and $1.7 \times 10^{12} 1 \mathrm{MeV} / n_{\mathrm{eq}}$ for Non-Ionizing Energy Loss (NIEL), which is the expected dose for 10 years of operation with LHC. The sensor size is about 
$1.5 \mathrm{~cm} \times 3 \mathrm{~cm}$ containing 512 rows and 1024 columns. The pixel pitch is $26.88 \mu \mathrm{m} \times 29.24 \mu \mathrm{m}$, which results in a spatial resolution of about $5 \mu \mathrm{m} \times 5 \mu \mathrm{m}$. The sensors are thinned down to $50 \mu \mathrm{m}$ for the IB and $100 \mu \mathrm{m}$ for the OB. The material budget of the ITS2 is reduced to $0.35 \% \mathrm{X}_{0}$ for the IB layers and $1.0 \% \mathrm{X}_{0}$ for the $\mathrm{OB}$ layers. The data of the fired pixels can be read out in serial at 1.2 Gbps for the IB sensors and 400 Mbps for the OB sensors.

The sensors are glued onto an aluminum-based Flexible Printed Circuit (FPC) to form a Hybrid Integrated Circuit (HIC). The HICs are then glued onto a carbon cold plate and a space frame to complete a stave. There are 48 staves for the IB and each stave hosts 9 sensors. For the OB, it consists 54 ML staves (112 chips per stave) and 90 OL staves (196 chips per stave). The detector covers a total surface of $10.3 \mathrm{~m}^{2}$ and contains about $12.5 \times 10^{9}$ pixels.

The sensor mass production and the series tests were completed in May 2019, and the HIC and stave production [4] were finished in November 2019. The qualified staves were then assembled into the half-layers. The verified half-layers were arranged together to form the four half-barrels: IB half-barrel Top (IBT), IB half-barrel Bottom (IBB), OB half-barrel Top (OBT), and OB halfbarrel Bottom (OBB). The assembly of the detector half-barrels was completed in December 2019. After the commissioning at the laboratory, the detector was successfully installed into the ALICE experiment in May 2021.

\section{Detector Commissioning and Performance}

The commissioning of the ITS2 mainly has two stages: the on-surface commissioning in a dedicated cleanroom at CERN and the on-site commissioning in the ALICE experiment. The on-surface commissioning started in July 2019 and finished in December 2020. The detector is being commissioned with other detectors in the ALICE experiment until February 2022.

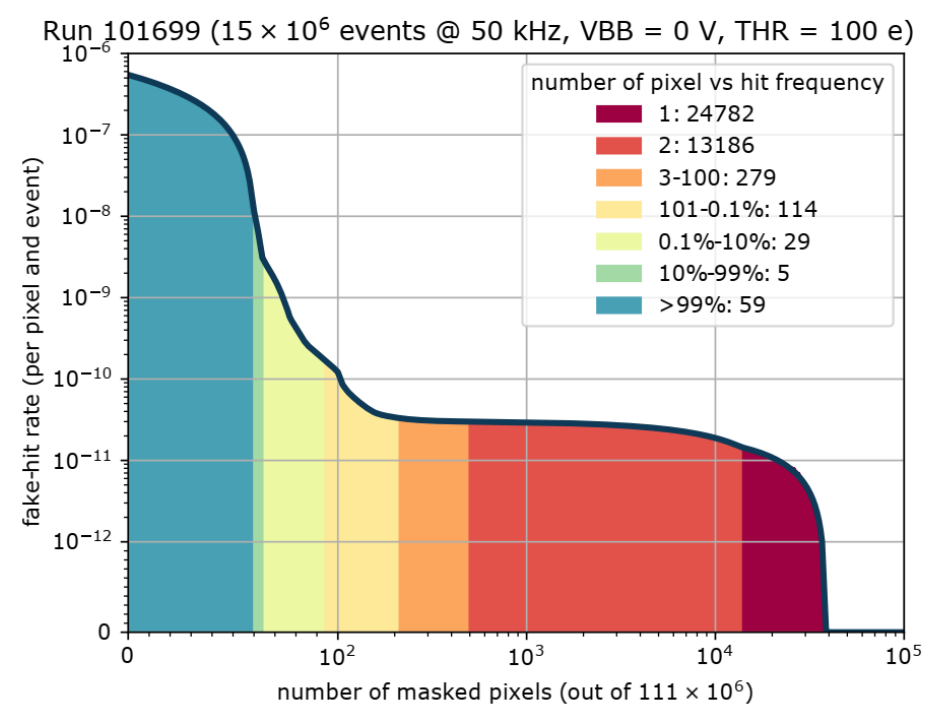

Figure 2: Fake-hit rate as a function of the number of masked noisy pixels for an IB half-layer. Sensor threshold tuned to 105 electrons, readout rate at $50 \mathrm{kHz}$ and the sensor back-bias voltage at $-3 \mathrm{~V}$. 


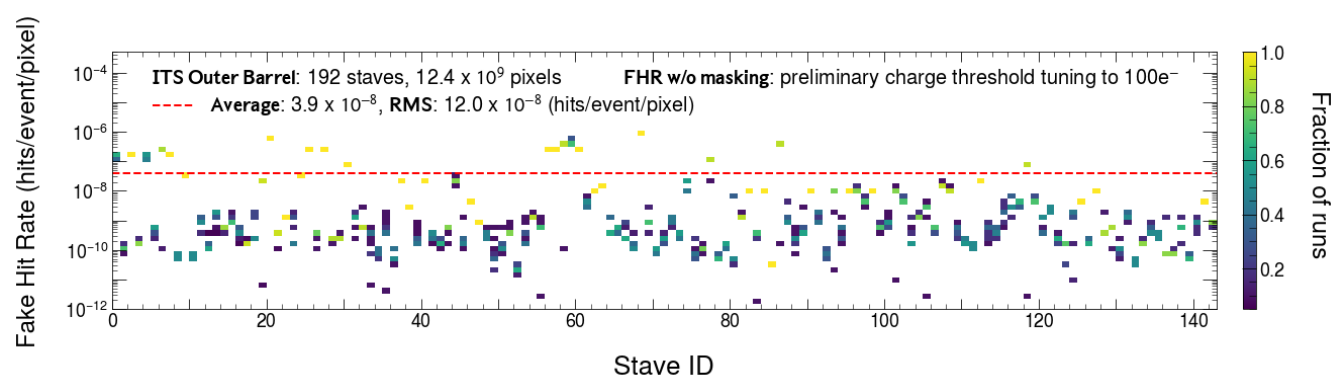

(a) Fake-hit rate for each stave before masking noisy pixels

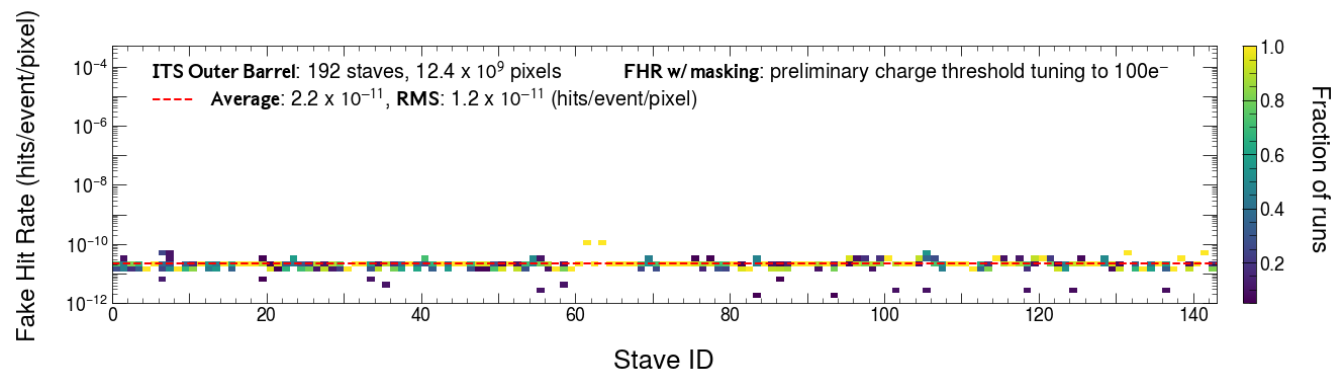

(b) Fake-hit rate for each stave after masking noisy pixels

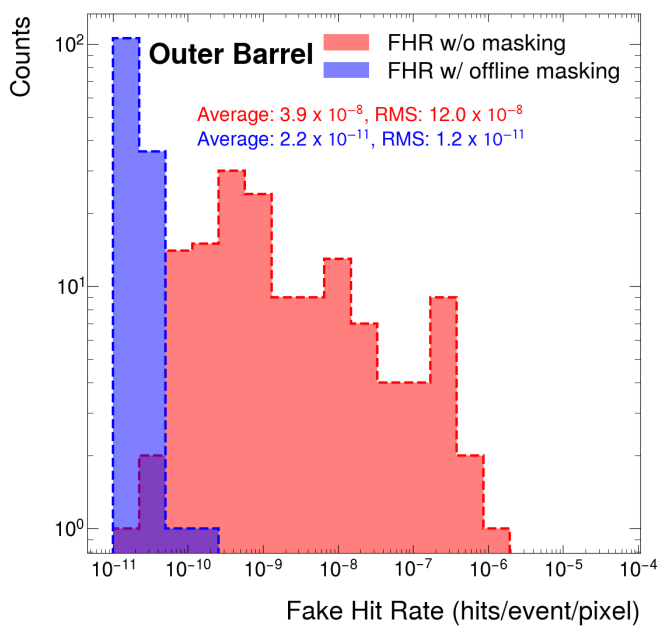

(c) Fake-hit rate distribution

Figure 3: Average fake-hit rate distribution for $O B$ staves before and after noisy pixel masking. Sensor threshold tuned to 100 electrons. Repeated 5 to 10 fake-hit rate runs for each stave to spot all the noisy pixels.

During the on-surface commissioning, the half-barrels were fully powered on and monitored to validate the long-term stability of the detector parameters. Different taking data runs with specific detector settings were also performed to investigate the performance of the detector. Some of the results on the detector fake-hit rate as well as the detection efficiency are discussed in this section.

The fake-hit rate was investigated by measuring the hits without any stimulation of the sensors. A set of fake-hit rate runs were carried out for both the IB and OB during the on-surface commissioning. The noise and the cosmic-rays are the main contributions for the fired pixels. The fake-hit rate as a function of the masked pixels for an inner half-barrel is shown in Fig. 2. A fake-hit 
rate of $10^{-10}$ hits/pixel/event is achieved after masking only a fraction of noisy pixels smaller than $2 \times 10^{-6}$ of the total pixels. For the OB, the fake-hit rate of each stave before and after noisy pixel masking and the corresponding distribution are shown in Fig. 3. A fake-hit rate of the order of $10^{-11}$ hits/pixel/event can be realized by masking up to $0.014 \%$ of the pixels for the worse case stave. One can see that the fake-hit rate is well below the required value of $10^{-6} \mathrm{hits} / \mathrm{pixel} / \mathrm{event}$ after masking the noisy pixels for the IB and OB.

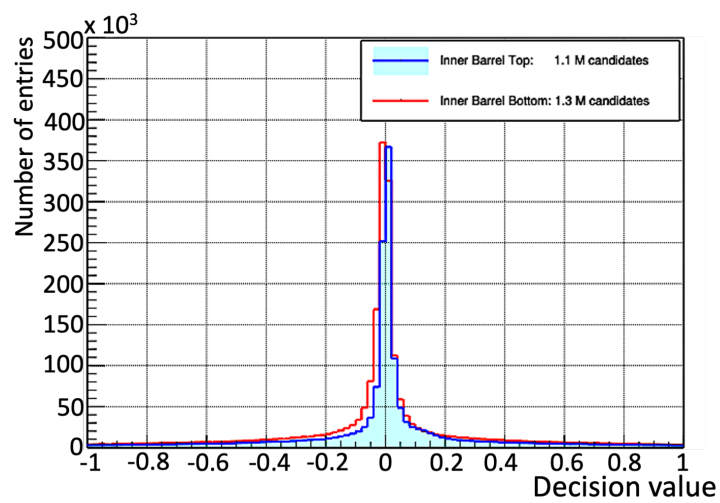

(a) IB

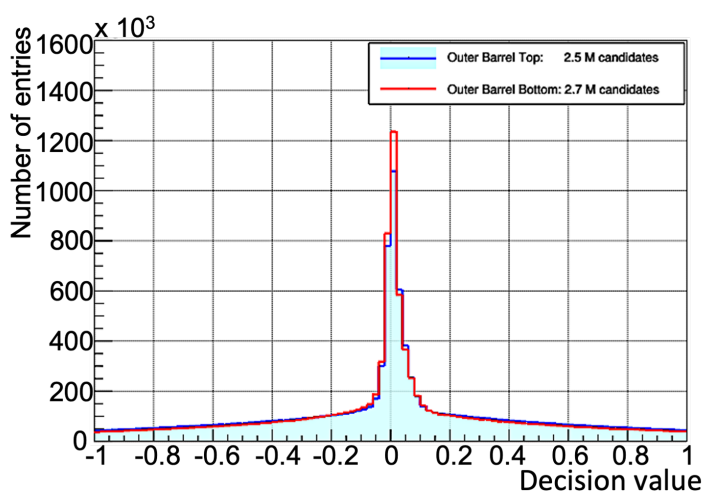

(b) $\mathrm{OB}$

Figure 4: Distributions of the decision value.

The alignment and detection efficiency are studied by reconstructing the cosmic-ray tracks from the fake-hit runs taken with the IB and OB. The track candidates were found by searching the cluster triplets which are located on the three layers of IB or OB. A decision value is defined as the vector product of the two vectors connecting the three clusters in the cluster triplet. The track is tagged as a potential cosmic event if the absolute decision value is smaller than 0.1. About $1.5 \times 10^{6}$ and $2.5 \times 10^{6}$ track candidates were reconstructed for the IB half-barrels and the OB half-barrels, respectively. The distributions of the decision value for the IB and OB are shown in Fig. 4. A preliminary analysis based on these track candidates reveals the residuals are in the order of $100 \mu \mathrm{m}$. Further alignment study will be done during the on-site commissioning.

The detection efficiency was also explored for the two OB half-barrels, which is defined as the fraction of the number of tracks detected within a search window, approximately the size of an ALPIDE sensor, and the total number of tracks expected within that search window. A track that hits at least three layers is extrapolated onto the fourth layer. The track is considered detected if a cluster is found within the search window on the fourth layer. The preliminary result of the detection efficiency for the half-layers on each half-barrel as a function of the angle of the searching window around the incidence angle of $\Delta \theta=90$ with respect to ground, $\delta \theta$, is shown in Fig. 5. One can see that a global detection efficiency of above $99.5 \%$ is reachable, which satisfies the requirement of $99 \%$ which is addressed in the technical design report.

\section{Summary}

The new Inner Tracking System, ITS2, is designed to improve the ALICE tracking capabilities, in particular at low transverse momentum. The ITS2 has been fully constructed and installed into 


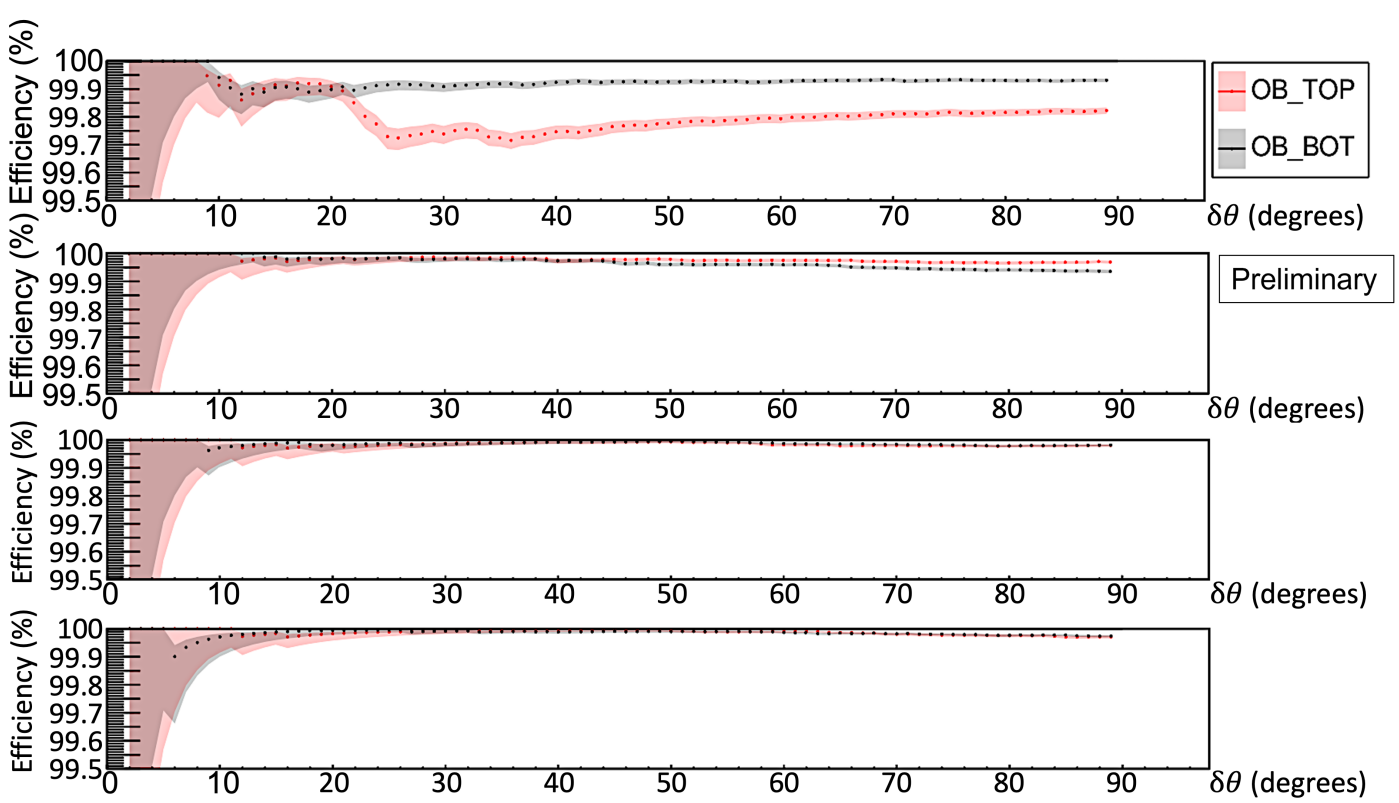

Figure 5: Detection efficiency for the OB half-layers (preliminary result).

the ALICE experiment. The preliminary results based on the on-surface commissioning reveal an excellent performance in terms of fake-hit rate and detection efficiency. A global commissioning together with the other ALICE sub-detectors is currently underway in order to get ready for the data taking of the first collisions provided by the LHC pilot beams in October 2021 and the LHC Run 3 in early 2022.

\section{References}

[1] The ALICE Collaboration, The ALICE experiment at the CERN LHC, JINST 3 (2008) S08002.

[2] The ALICE Collaboration, Technical Design Report for the Upgrade of the ALICE Inner Tracking System, J. Phys. G 41 (2014) 087002

[3] M. Mager, ALPIDE, the Monolithic Active Pixel Sensor for the ALICE ITS upgrade, Nucl. Instrum. Methods Phys. Res. A 824 (2014) 434 - 438

[4] G. Contin, The MAPS-based ITS Upgrade for ALICE, PoS Vertex2019 (2020) 003 